\title{
Analyses of DNA methylation involved in the activation of nuclear karyopherin alpha 2 leading to the progression and prognostic significance across human breast cancer
}

\section{Xiangrong Cui}

Reproductive Medicine Center, Children's Hospital of Shanxi and Women Health Center of Shanxi, Affiliated of Shanxi Medical University, Taiyuan, 030001 (China)

Hongping Liang

Clinical laboratory, Shanxi Prov. People's Hospital, Affiliated of Shanxi Medical University, Taiyuan, 030001 (China)

\section{Chonghua Hao}

Clinical laboratory, Shanxi Prov. People's Hospital, Affiliated of Shanxi Medical University, Taiyuan, 030001 (China)

\section{Kai Huo}

Department of breast surgery, Shanxi Cancer Hospital, 030000 (China)

\section{Xuan Jing ( $\nabla$ jx05070103@163.com )}

Shanxi Provincial Peoples Hospital

\section{Research}

Keywords: KPNA2, Breast cancer, Prognosis, Methylation, TPX2

Posted Date: February 26th, 2020

DOI: https://doi.org/10.21203/rs.2.24589/v1

License: (c) (1) This work is licensed under a Creative Commons Attribution 4.0 International License. Read Full License 


\section{Abstract}

Background: Karyopherin alpha 2 (KPNA2) is a nuclear import factor that plays a crucial role in nucleocytoplasmic transport, as well as cell proliferation, migration and invasion in several cancers. However, the roles of KPNA2 in breast cancer as well as the underlying molecular mechanisms have not been elucidated.

Methods: To evaluate gene expression alterations during breast carcinogenesis, KPNA2 expression was analyzed using the Gene Expression Profiling Interactive Analysis and Oncomine analyses. The correlation between methylation and expression was analyzed using the MEXPRESS tool, UALCAN cancer database, and cBioPortal browser. Then, the expression and prognostic value of KPNA2 was investigated by our own breast cancer samples using RT-PCR. KPNA2 methylation level was detected by methylationspecific PCR.

Results: We obtained the following important results. (1) KPNA2 expression was significantly higher in breast cancer than normal samples and regulated by aberrant DNA hypomethylation of promoter region. (2) Among patients with breast cancer, those with higher KPNA2 expression had a lower survival rate. (3) The major mutation type of KPNA2 in breast cancer samples was missense mutation. (4) Homer1 was able to promote breast cancer progression may be through up-regulating TPX2 expression.

Conclusions: Our findings suggest that aberrant DNA hypomethylation of promoter regions contributes to the aberrant expression of KPNA2 in breast cancer, which might be a potential indicator of poor prognosis.

\section{Introduction}

Breast cancer (BC) is the most frequent malignant tumor in women and account for $24.2 \%$ of female new cases worldwide ( 8.6 million new cases), with about $15.0 \%$ associated deaths globally (4.2 million deaths) in 2018 according to Global Cancer Statistics 2018 [1]. Although the progression of modern medical technology has advanced the therapeutic effect of breast cancer, it remains the main cancerrelated cause of female deaths [2-5]. Therefore, the progress in molecular diagnostic and recognition of prognostic value biomarkers in patients are desired in the medical field.

Karyopherin alpha 2 (KPNA2; also known as importina1 or RAG cohort 1), is one of the seven members of karyopherin alpha family, which plays a crucial role in nucleocytoplasmic transport [5-9]. KPNA2 cooperates with nuclear receptor importin- $\beta$ and mediates numerous nuclear translocations of target proteins guided cargoproteins through the nuclear pore complex $[9,10]$. Previous studies have reported a significant amount of KPNA2 protein expressed is observed in various types of cancer, including breast cancer, gastric cancer, lung cancer, and prostate cancer, suggesting that KPNA2 may play some roles in these tumors [10-13]. Further analysis also demonstrated an independent negative correlation between nuclear KPNA2 protein expression in the primary tumor and overall survival (OS) of breast cancer patients 
$[14,15]$. However, the precise role of KPNA2 in breast cancer and the molecular mechanisms underlying its effects have not been elucidated.

In the current study, we hypothesized that KPNA2 might be a promising candidate as diagnostic and prognostic marker for breast cancer. To test this hypothesis, we performed a bioinformatics method to determine the expression and prognostic value of KPNA2 in breast cancer overall and its subtypes. Furthermore, we identified the mutation and methylation status of KPNA2 in breast cancer to investigate the molecular mechanisms for the effect of increased KPNA2 expression on breast cancer. Our results demonstrated that relative expression of KPNA2 was downregulated and KPNA2 was hypomethylated in breast cancer tissues and cells, which will contribute to the development and optimization of novel diagnosis and therapeutics for breast cancer.

\section{Methods}

\section{Clinical samples}

This study enrolled a consecutive series of 30 patients with breast cancer from the middle area of China. As a control, we used 20 non-tumoral-adjacent tissues. Frozen tissues were collected at the Department of Breast Surgery from Shanxi Tumour Hospital (China). The patients ranged in age from 34 to 75 years with a mean age of 48.62 years. Fresh tissues were frozen in liquid nitrogen within 5 min after excision and transferred to a $-80^{\circ} \mathrm{C}$ freezer. The entire experiments have already obtained the approval of the Ethics Committee of Shanxi Medical niversity (Ethical code: 201922021).

\section{Cell culture}

Human breast cancer cell line (MCF7) was obtained from the American Type Culture Collection (ATCC, Rockville, MD, USA). MCF7 cells were maintained in DMEM/F12 medium supplemented 10\% FBS (fetal bovine serum) (both from Gibco; Thermo Fisher Scientific, Inc., Waltham, MA, USA), $100 \mathrm{U} / \mathrm{ml}$ penicillin and $100 \mathrm{mg} / \mathrm{ml}$ streptomycin. Culture plates were maintained at $37{ }^{\circ} \mathrm{C}$ in a $5 \% \mathrm{CO}_{2}$ humidified atmosphere.

\section{DNA methylation inhibitor treatment}

The same numbers of cells MCF7 cells were cultured in 6-well plates and incubated at $37^{\circ} \mathrm{C}$ overnight. Cells were treated with 1,5 , or $10 \mu \mathrm{M}$ Decitabine (Selleck, Houston, TX, USA) or vehicle (DMSO) and incubated for 72 with the purpose of mRNA extraction. The cell culture medium was refreshed daily.

\section{Quantitative real-time PCR (qRT-PCR)}

RNA extraction kit (BioTeke Corporation, Beijing, China) was employed to extract total RNAs. Prime Script $^{\text {TM }}$ RT Master Mix kit (Takara Botechnology Co., Ltd., Dalian, China )was conducted to reverse transcribe the extracted RNA into complementary DNA. The qRT-PCR was carried out following SYBR ${ }^{8}$ Premix Ex Taq Kit (Takara Bio, Inc., Tokyo, Japan). For PCR amplification, glyceraldehyde -phosphate dehydrogenase (GAPDH) was employed as a suitable internal control. The results were recorded when the 
cycle was finished. The primers used were as follows: KPNA2, forward, 5'-ATTGCAGGTGATGGCTCAGT-3' and reverse, 5'-CTGCTCAACAGCATC TATCG-3'; GAPDH, forward, 5'-ACCACAGTCCATGCCATCAC-3'; and reverse, 5'-TCCACCA CCCTGTTGCTGTA-3'. Relative gene expression was calculated with the $2^{-\triangle \triangle C t}$ method, and Step One Software v2.1 was used to evaluate the results.

\section{Methylation specific PCR}

The extraction of genomic DNA from cells was performed by the use of a DNeasy Tissue Kit (Qiagen, Hilden, Germany) according to the manufacturer's protocol. DNA samples were PCR-amplified and fragmented in a volume of $20 \mu \mathrm{l}$ comprised of $0.25 \mu \mathrm{l}$ of Hot-StarTaq Master Mix, $0.5 \mu \mathrm{M}$ of paired primer, and $2 \mu \mathrm{g}$ of bisulfite-treated DNA template. The agarose gel (3\%) containing GelRed ${ }^{\text {TM }}$ Nucleic Acid Gel Stain (10,000x; Biotium, CA) was used to separate MSP products in a $20 \mu \mathrm{l}$ volume after amplification. DNA-free water was served as negative control. The primers used were as follows: forward M primer, $5^{\prime}-$ AAATACGAACGGTTTAGGGAATC - 3' and reverse M primer, 5'- ACATCGCGAAATAAAAAAACG - 3'; forward U primer, 5'-GGAAAT ATGAATGGTTTAGGGAATT-3', reverse U primer, 5'-ATAACATCACAAAATAAAAAAACAA C-3'.

\section{Gene Expression Profiling Interactive Analysis}

Differential expressions of KPNA2 in breast invasive carcinoma tissues were assessed by GEPIA2 (Gene Expression Profiling Interactive Analysis; http://gepia2.cancer-pku.cn), an online database with fast and customizable features based on TCGA (The Cancer Genome Atlas) [16]. Here, GEPIA database containing 1085 breast invasive carcinoma tissues and 112 normal mammary tissues were downloaded for analyses. Gene expression data of the RNA-seq datasets were transformed to $\log _{2}$ (transcript count per million [TPM] + 1).

\section{Oncomine database analysis}

KPNA2 gene expression levels in normal or malignant human tissues were obtained from the Oncomine Cancer Microarray database (http://www.oncomine.org), a web-based data mining platform for collecting, standardizing, analyzing and providing cancer microarray information [17].

\section{cBioPortal database analysis}

cBioPortal (The cBio Cancer Genomics Portal) database is a publicly accessible online database (http://www.cbioportal.org/) [18, 19], which provides visualization and analysis tool for more than 715 datasets and 86,733 samples. The term "KPNA2" was used to search the cBioPortal database and The Breast Invasive Carcinoma (TCGA, Cell 2015, $n=818$ ) cohort was utilized. The search parameters included mutations, putative copy-number, and methylation alterations from GISTIC.

\section{COSMIC analysis}

The COSMIC (Catalogue of Somatic Mutations in Cancer) database (http://cancer.sanger.ac.uk), an online accessible network tool was performed to analysis mutations in KPNA2. An overview of the 
distribution of mutations and substitution types on the codogenic strand in breast cancer specimens was perfomed, and the results are drawn in a pie chart [20]

\section{Breast Cancer Gene-Expression Miner v4.4}

bcGenExMiner v4.4 (Breast Cancer Gene-Expression Miner v4.4), a web-based tool, was performed to analysis the KPNA2 expression in breast cancer [21]. Correlations between KNPA2 and the TPX2 were analyzed using the bcGenExMiner v4.4 correlation module.

\section{Kaplan-Meier plotter analysis}

Kaplan-Meier Plotter (http://kmplot.com/analysis/) is an online database of published microarray datasets that assess the effect of 54, 675 genes on survival using 10, 461 cancer samples (6, 234 breast, 2, 190 ovarian, 3, 452 lung, and 1, 440 gastric cancer). We used the Kaplan-Meier plotter to assess the correlation between overall survival, relapse free survival, distant metastasis free survival, postprogression survival and KPNA2 mRNA expression in patients with breast cancer. The hazard ratio (HR) with $95 \%$ confidence intervals (Cl) and log rank p-value were also computed.

\section{MEXPRESS tool}

The MEXPRESS tool (http://mexpress.be/), a web tool for the visualization and interpretation of TCGA data, offers clinical researchers an easy way to access TCGA expression (normalized RNASeqV2 value), DNA methylation, and clinical data, as well as the relationship between them for one signale in the specific tumor type. In MEXPRESS tool, it executed the Pearson correlation to evaluate the difference between expression value and methylation level. In our research, the KNPA2 expression and methylation status in breast cancer were assessed using the MEXPRESS tool.

\section{UALCAN cancer database}

UALCAN (http://ualcan.path.uab.edu/) is a comprehensive, user-friendly, and interactive online portal for analyzing cancer OMICS data, which provides protein expression analysis option using data from TCGA dataset [22-24]. We evaluated the expression and methylation level of KPNA2 in breast cancer by UALCAN analysis.

\section{Results}

\section{KPNA2 transcript expression status in human breast cancer}

The expression profile of KPNA2 was identified using Gene Expression Profiling Interactive Analysis. GEPIA data showed that the expression level of KPNA2 was significantly higher in BLCA (bladder urothelial carcinoma), BRCA (breast cancer), CESC (cervical squamous cell carcinoma and endocervical adenocarcinoma), COAD (colon adenocarcinoma), DLBC (lymphoid neoplasm diffuse large B-cell lymphoma), ESCA (esophageal carcinoma), GBM (glioblastoma multiforme), HNSC (head and neck squamous cell carcinoma), LIHC (liver hepatocellular carcinoma), LUAD (lung adenocarcinoma), LUSC 
(lung squamous cell carcinoma), OV (ovarian serous cystadenocarcinoma), PAAD (pancreatic adenocarcinoma), READ (rectum adenocarcinoma), SKCM (skin cutaneous melanoma), STAD (stomach adenocarcinoma), THYM (thymoma), UCEC (uterine corpus endometrial carcinoma), UCS (uterine carcinosarcoma) (Fig. 1). To further confirm this result, the Oncomine database was performed to analysis the expression profile of KPNA2. Elevated mRNA expression of KPNA2 was identified in various human tumors, including bladder cancer, brain and CNS cancer, breast cancer, cervical cancer, colorectal cancer, esophageal cancer, gastric cancer, head and neck cancer, kidney cancer, liver cancer, lung cancer, melanoma, myeloma, ovarian cancer, pancreatic cancer, and sarcoma (Fig. 2A). KPNA2 expression was significantly higher in ductal breast carcinoma, male breast carcinoma, invasive ductal breast carcinoma, invasive breast carcinoma, invasive lobular breast carcinoma, ductal breast carcinoma in situ, and medullary breast carcinoma than in normal samples (Table 1, Fig. 2B).

Table 1

KPNA2 expression in breast cancer

\begin{tabular}{|c|c|c|c|c|c|c|}
\hline Cancer subtype & p-value & $\begin{array}{l}\text { Fold } \\
\text { change }\end{array}$ & t-test & $\begin{array}{l}\text { Rank } \\
(\%)\end{array}$ & Sample & Reference \\
\hline Ductal breast carcinoma & $\begin{array}{l}6.62 \mathrm{E}- \\
18\end{array}$ & 5.334 & 16.133 & 3 & 47 & [36] \\
\hline Male Breast Carcinoma & $\begin{array}{l}1.84 \mathrm{E}- \\
31\end{array}$ & 4.718 & 33.375 & 12 & 64 & TCGA \\
\hline $\begin{array}{l}\text { Invasive Ductal Breast } \\
\text { Carcinoma }\end{array}$ & $\begin{array}{l}5.54 \mathrm{E}- \\
47\end{array}$ & 3.208 & 22.121 & 74 & 450 & TCGA \\
\hline Invasive Breast Carcinoma & $\begin{array}{l}4.35 \mathrm{E}- \\
25\end{array}$ & 2.721 & 12.945 & 225 & 137 & TCGA \\
\hline $\begin{array}{l}\text { Invasive Lobular Breast } \\
\text { Carcinoma }\end{array}$ & $\begin{array}{l}1.58 \mathrm{E}- \\
11\end{array}$ & 2.057 & 8.226 & 715 & 97 & TCGA \\
\hline $\begin{array}{l}\text { Ductal Breast Carcinoma in } \\
\text { Situ }\end{array}$ & 8.67E-6 & 2.099 & 5.609 & 118 & 25 & [37] \\
\hline $\begin{array}{l}\text { Invasive Ductal Breast } \\
\text { Carcinoma }\end{array}$ & $\begin{array}{l}9.54 \mathrm{E}- \\
81\end{array}$ & 2.224 & 27.698 & 386 & 2136 & [38] \\
\hline Breast Carcinoma & 4.00E-5 & 2.078 & 5.497 & 852 & 158 & [38] \\
\hline Invasive Breast Carcinoma & $1.99 \mathrm{E}-5$ & 2.142 & 5.164 & 1177 & 165 & [38] \\
\hline Medullary Breast Carcinoma & $1.23 \mathrm{E}-8$ & 2.594 & 7.248 & 1292 & 176 & [38] \\
\hline
\end{tabular}

\section{KPNA2 mutations in breast cancer}

The pie chart in Fig. 3A generated using COSMIC summarizes the observed mutation types, including nonsense substitutions, missense substitutions, synonymous substitutions, in-frame insertions, frameshift insertions, in-frame deletions, frameshift deletions, and complex mutations. Mutation of in 
breast cancer samples mainly is missense substitution (30.00\%). Furthermore, KPNA2 mutations in breast cancer samples were $16.67 \% \mathrm{~A}>\mathrm{G}, 33.33 \% \mathrm{C}>\mathrm{T}, 33.33 \% \mathrm{G}>\mathrm{A}$, and $16.67 \% \mathrm{G}>\mathrm{T}$. As determined using cBioPortal, the KPNA2 mutation frequency was less than $1.5 \%$ in patients with breast cancer. (Fig. 3B).

\section{Genetic alterations in KPNA2 and clinicopathological parameters}

We analyzed the expression profile of KPNA2 across PAM50 breast cancer subtypes in 5861 patients using bcGenExMiner v4.4 web-tool based on clinical-pathological parameters. Regarding age, KPNA2 mRNA expression were remarkably higher in patients $\leq 51$ years old than in patients $>51$ years old. Furthermore, the expression of KNPA2 decreased significantly with age (Table 2, Fig. 4). ER (estrogen receptor) and PR (progesterone receptor) status were negatively correlated with KPNA2 expression, while HER-2 (human epidermal growth factor receptor-2) and nodal status were positively correlated with KPNA2 expression (Table 2, Fig. 4). Triple-negative breast cancer (TNBC) is negative for ER, PR, and HER2. KPNA2 mRNA expression was significantly upregulated in patients with TBNC $(p<0.0001)$ compared with that in the group without TBNC $(p<0.0001)$ (Table 2, Fig. 4). Furthermore, patients with positive basal-like characteristics and mutated P53 status exhibited significantly higher KPNA2 expression than that in patients without basal-like characteristics $(p<0.0001)$ (Table 2, Fig. 4). A more advanced Scarff Bloom \& Richardson grade status (SBR) and Nottingham Prognostic Index grade status (NPI) grades were associated with higher KPNA2 expression (Table 2). 
Table 2

The relationship between mRNA expression of KPNA2 and clinicopathological parameters of breast carcinoma

\begin{tabular}{|c|c|c|c|}
\hline Variables & No.* & mRNA & $\mathrm{p}$-value \\
\hline \multicolumn{4}{|l|}{ Age } \\
\hline$\leq 51$ & 28000 & $\uparrow$ & $<0.0001$ \\
\hline$>51$ & 4634 & - & \\
\hline \multicolumn{4}{|l|}{ Age } \\
\hline$[21 ; 40]$ & 795 & $\uparrow$ & $<0.0001$ \\
\hline ]40;70[ & 5212 & $\uparrow$ & $<0.01$ \\
\hline$[70 ; 97]$ & 1427 & - & \\
\hline \multicolumn{4}{|c|}{ Nodal status } \\
\hline- & 4358 & - & 0.0169 \\
\hline+ & 3460 & $\uparrow$ & \\
\hline \multicolumn{4}{|l|}{ ER (IHC) } \\
\hline- & 2226 & - & $<0.0001$ \\
\hline+ & 6262 & $\downarrow$ & \\
\hline \multicolumn{4}{|l|}{ PR (IHC) } \\
\hline- & 1427 & - & $<0.0001$ \\
\hline+ & 1994 & $\downarrow$ & \\
\hline \multicolumn{4}{|l|}{ HER2 (IHC) } \\
\hline- & 2387 & - & $<0.0001$ \\
\hline+ & 436 & $\uparrow$ & \\
\hline \multicolumn{4}{|c|}{ Triple-negative Status } \\
\hline Not & 6477 & - & $<0.0001$ \\
\hline TNBC & 572 & $\uparrow$ & \\
\hline \multicolumn{4}{|c|}{ Basal-like Status } \\
\hline Not & 7120 & - & $<0.0001$ \\
\hline Base-like & 1838 & $\uparrow$ & \\
\hline
\end{tabular}




\begin{tabular}{|lccc|}
\hline Variables & No.* & mRNA & p-value \\
\hline Basal-like \& TNBC Status & & & \\
\hline Not & 5744 & - & $<0.0001$ \\
\hline Basal-like \& TNBC & 406 & $\uparrow$ & \\
\hline P53 status & & & \\
\hline Wild type & 638 & - & $<0.0001$ \\
\hline Mutated & 284 & $\uparrow$ & \\
\hline SBR Status & & & \\
\hline SBR1 & 864 & - & \\
\hline SBR2 & 2907 & $\uparrow$ & $<0.0001$ \\
\hline SBR3 & 2906 & $\uparrow$ & $<0.0001$ \\
\hline NPI Status & & & \\
\hline NPI1 & 1187 & - & \\
\hline NPI2 & 2084 & $\uparrow$ & $<0.0001$ \\
\hline NPI3 & 686 & $\uparrow$ & $<0.0001$ \\
\hline
\end{tabular}

\section{Co-expression of KPNA2 gene}

To investigate the regulatory mechanisms underlying the role of KPNA2 in breast cancer, data mining was performed for a breast cancer cohort using cBioPortal. TPX2 (Targeting protein for Xenopus kinesin-like protein 2) is a highly correlated gene (Fig. 5A); it drives proliferation, tumorigenicity and metastasis of various tumors through regulating cell cycle and multiple signaling pathways that promote tumor proliferation. A regression analysis using cBioPortal revealed that KPNA2 and TPX2 levels are highly correlated, which demonstrated that KPNA2 may be related to TPX2 pathway in breast cancer (Fig. 5B).

\section{Relationship of KPNA2 expression and prognosis in breast cacners}

To explore whether the expression level of KPNA2 has predictive value for breast cancer prognosis, we used the online survival analysis software Kaplan-Meier plotter (Fig. 6). It was determined that a high expression of KPNA2 mRNA was significantly related with OS (overall survival), RFS (relapse free survival), DMFS (distant metastasis free survival), and PPS (post-progression survival). Our own results demonstrated the upregulation of KPNA2 mRNA expression and worse probabilities of survival in breast cacner (Fig. 7) 


\section{KPNA2 expression is upregulated by hypomethylation in breast tumors}

Using the MEXPRESS browser, we generated the methylation of KPNA2 using 13 probes distributed in different regions of the gene (the localization of each probe is presented in the figure, and those localized in the promoter region are highlighted in dark blue). All regions analyzed revealed a negative correlation with respect to KPNA2 gene expression (Pearson's correlation coefficients for each probe are indicated on the right in Fig. 8, suggesting that KPNA2 methylation silences gene expression.). Furthermore, we analyzed the expression and promoter methylation level of KPNA2 using UALCAN tool from TCGA dataset. We found the methylation level of KPNA2 in breast cancer was significantly lower than normal samples $(p=3.49 \mathrm{E}-12)$. Subsequently, we evaluated he expression and methylation level of KPNA2 in breast cancer by cBioPortal tool. We further confirmed a negative correlation with respect to KPNA2 gene expression, suggesting that KPNA2 methylation silences gene expression (Fig. 9).

\section{Decitabine caused demethylation and the activation of KPNA2 in breast cancer}

The effects of epigenetic agents on breast cancer progression were investigated using the minimum effective dose which was $1 \mu \mathrm{M}$ for decitabine (Fig. 10A). After decitabine treatment, the degree of demethylation was lower in the MCF7 cell line than that in untreated cells (Fig. 10B). The result of the relative mRNA expression showed the upregulated by the treatment of decitabine against its expression in control cells (Fig. 10C). Furthermore, cell proliferation was promoted by the decitabine treatment (Fig. 10D).

\section{Discussion}

KPNA2, an adaptor protein for nuclear receptor importin- $\beta$, mediates numerous nuclear translocations of macromolecules by classical nuclear localization singal through the nuclear pore complex [25-27]. KPNA2 has been first descripted in matched ductal carcinoma in situ (DCIS) and invasive lesions of the breast [28]. Furthermore, KPNA2 may be the primary determinant for transcription factors transporting and transcriptional activity in various cancers including breast cancer, melanoma, liver cancer, and lung cancer, and therefore is probably related to the cancer cell growth and invasion [15, 29-31]. These findings suggest that high KPNA2 expression in patients with breast may be related with worse prognosis.

To further investigate the role of KPNA2 in the development and proliferation of breast cancer, we analyzed extensive gene expression data with well-defined parameters in breast cancer and nomal samples. Using GEPIA and Oncomine, we found that the expression level of KPNA2 is significantly higher in breast cancer tissues than in normal samples. Using Oncomine, we further determined that KPNA2 is in ductal breast carcinoma, male breast carcinoma, invasive ductal breast carcinoma, invasive breast 
carcinoma, invasive lobular breast carcinoma, ductal breast carcinoma in situ, and medullary breast carcinoma than in normal samples. Furthermore, we analyzed the expression profile of KPNA2 across PAM50 breast cancer using bcGenExMiner v4.4 web-too, we demonstrated that age, ER and PR status were negatively correlated with KPNA2 expression, while basal-like, mutated P53, HER-2, SBR, NPI, and nodal status were positively correlated with KPNA2 expression.

Somatically acquired inherited, epigenetic, transcriptomic, and proteomic alterations are the major alterations occur in specific genomic regions, which could lead inhibitory or carcinogenic roles [32-34]. Therefore, the frequencies of alterations and mutations in KPNA2 were analyzed using the COSMIC and cBioPortal databases. The major mutation type in KPNA2 was missense substitution. However, a low KPNA2 alteration frequency was observed in breast cancer. We investigated the mechanisms underlying KPNA2 dysregulation.

DNA methylation is an early event in the process of tumorigenesis and gradually enhances during tumor progression [35]. Therefore, DNA methylation of precancerous lesions or early tumors is the most promising method for early diagnosis and prognosis of cancer. By examining its DNA methylation status through MEXPRESS web-tool, we observed that aberrant DAN hypomethylation of promoter regions is one of the mechanisms underlying the aberrant expression of KPNA2 in breast cancer. By UALCAN and cBioPortal analyses further indicated that the methylation level of KPNA2 in breast cancer was significantly lower than normal samples and negative correlation with respect to KPNA2 gene expression. Interestingly, when methylation was inhibited, the expression of kpna2 was significantly increased and the proliferation ability was significantly enhanced.

\section{Conclusions}

In summary, KPNA2 is highly expressed in breast cancer and is independent risk factor for poor prognosis in breast cancer patients. Furthermore, KPNA2 has a role in malignancy mainly through the TPX2 signaling pathway, which is a potential therapeutic target for breast cancer. Finally, our results suggest that aberrant DNA hypomethylation of promoter regions contributes to the aberrant expression of KPNA2 in breast cancer and may be an indicator of poor prognosis.

\section{Abbreviations}

KPNA2: Karyopherin alpha 2; GAPDH: glyceraldehyde - phosphate dehydrogenase; DCIS: ductal carcinoma in situ; TPX2: Targeting protein for Xenopus kinesin-like protein 2; BLCA: bladder urothelial carcinoma; BRCA: breast cancer; CESC: cervical squamous cell carcinoma and endocervical adenocarcinoma; COAD: colon adenocarcinoma; DLBC: lymphoid neoplasm diffuse large B-cell lymphoma; ESCA: esophageal carcinoma; GBM: glioblastoma multiforme; HNSC: head and neck squamous cell carcinoma; LIHC: liver hepatocellular carcinoma; LUAD: lung adenocarcinoma; LUSC: lung squamous cell carcinoma; OV: ovarian serous cystadenocarcinoma; PAAD: pancreatic adenocarcinoma; READ: rectum adenocarcinoma; SKCM: skin cutaneous melanoma; STAD: stomach adenocarcinoma; 
THYM: thymoma; UCEC: uterine corpus endometrial carcinoma; UCS: uterine carcinosarcoma; bcGenExMiner v4.4: Breast Cancer Gene-Expression Miner v4.4; OS: overall survival; RFS: relapse free survival; DMFS: distant metastasis free survival; PPS: post-progression survival.

\section{Declarations}

\section{Acknowledgements}

Not applicable.

\section{Funding}

This study was supported by the Scientific Research Project of Shanxi Provincial Department of Health (grant no. 201601070), Initial Scientific Research Fund of PhD in Shanxi Provincial People's Hospital (grant no. b201635), Natural Science Foundation of Shanxi (grant no. 201901D211519), and Natural Science Foundation of Shanxi (grant no. 201901D211546), Research Project Supported by Shanxi Scholarship Counicle of China (grant no. HGKY2019092).

\section{Availability of data and materials}

The datasets generated for this study are available on request to the corresponding author.

\section{Author details}

1. Reproductive Medicine Center, Children's Hospital of Shanxi and Women Health Center of Shanxi, Affiliated of Shanxi Medical University, Taiyuan, 030001 (China); 2. Clinical laboratory, Shanxi Prov. People's Hospital, Affiliated of Shanxi Medical University, Taiyuan, 030001 (China); 3. Department of breast surgery, Shanxi Cancer Hospital, 030000 (China).

\section{Authors' contributions}

Xuan Jing designed the study, supported data analysis and wrote the paper. Xiangrong Cui was involved in the study design and supported data analysis. Hongping Liang and Chonghua Hao participated in the design of the study. Kai Huo is the senior investigator who designed research and wrote the paper. All authors read and approved the final manuscript.

\section{Ethics approval and consent to participate}

Informed consent was obtained from each participant. The entire experiments have already obtained the approval of the Ethics Committee of Shanxi Medical niversity (Ethical code: 201922021).

\section{Consent for publication}

All the authors in this paper consent to publication of the work. 
Competing interests

The authors declare that they have no competing interests

\section{References}

1. Bray F, Ferlay J, Soerjomataram I, Siegel RL, Torre LA, Jemal A: Global cancer statistics 2018: GLOBOCAN estimates of incidence and mortality worldwide for 36 cancers in 185 countries. CA: a cancer journal for clinicians 2018, 68(6):394-424.

2. Qin Y, Liu Y, Zhang X, Zhao S, Zhong H, Huang J, Yu J: Contrastenhanced spectral mammography: A potential exclusion diagnosis modality in dense breast patients. Cancer medicine 2020.

3. Khosrow-Khavar F, Filion KB, Bouganim N, Suissa S, Azoulay L: Aromatase Inhibitors and the Risk of Cardiovascular Outcomes in Women With Breast Cancer: A Population-Based Cohort Study. Circulation 2020, 141(7):549-559.

4. Hassett MJ, Somerfield MR, Baker ER, Cardoso F, Kansal KJ, Kwait DC, Plichta JK, Ricker C, Roshal A, Ruddy KJ et al: Management of Male Breast Cancer: ASCO Guideline. Journal of clinical oncology : official journal of the American Society of Clinical Oncology 2020:JC01903120.

5. Huang JX, Wu YC, Cheng YY, Wang CL, Yu CJ: IRF1 Negatively Regulates Oncogenic KPNA2 Expression Under Growth Stimulation and Hypoxia in Lung Cancer Cells. OncoTargets and therapy 2019, 12:11475-11486.

6. Han Y, Wang X: The emerging roles of KPNA2 in cancer. Life sciences 2020, 241:117140.

7. Lin F, Gao L, Su Z, Cao X, Zhan Y, Li Y, Zhang B: Knockdown of KPNA2 inhibits autophagy in oral squamous cell carcinoma cell lines by blocking p53 nuclear translocation. Oncology reports 2018, 40(1):179-194.

8. Ma S, Zhao X: KPNA2 is a promising biomarker candidate for esophageal squamous cell carcinoma and correlates with cell proliferation. Oncology reports 2014, 32(4):1631-1637.

9. Tachita T, Kinoshita S, Ri M, Aoki S, Asano A, Kanamori T, Yoshida T, Totani H, Ito A, Kusumoto S et al: Expression, mutation, and methylation of crbn-pathway genes at pre- and post-lenalidomide treatment in multiple myeloma. Cancer science 2020.

10. Song KH, Jung SY, Park JI, Ahn J, Park JK, Um HD, Park IC, Hwang SG, Ha H, Song JY: Inhibition of Karyopherin-alpha2 Augments Radiation-Induced Cell Death by Perturbing BRCA1-Mediated DNA Repair. International journal of molecular sciences 2019, 20(11).

11. Wang $\mathrm{Cl}$, Chen YY, Wang $\mathrm{CL}$, Yu JS, Chang YS, Yu CJ: mTOR regulates proteasomal degradation and Dp1/E2F1- mediated transcription of KPNA2 in lung cancer cells. Oncotarget 2016, 7(18):2543225442.

12. Tsai MM, Huang HW, Wang CS, Lee KF, Tsai CY, Lu PH, Chi HC, Lin YH, Kuo LM, Lin KH: MicroRNA26b inhibits tumor metastasis by targeting the KPNA2/c-jun pathway in human gastric cancer. Oncotarget 2016, 7(26):39511-39526. 
13. Grupp K, Boumesli R, Tsourlakis MC, Koop C, Wilczak W, Adam M, Sauter G, Simon R, Izbicki JR, Graefen $\mathrm{M}$ et al: The prognostic impact of high Nijmegen breakage syndrome (NBS1) gene expression in ERG-negative prostate cancers lacking PTEN deletion is driven by KPNA2 expression. International journal of cancer 2014, 135(6):1399-1407.

14. Lu Y, Xiao L, Liu Y, Wang H, Li H, Zhou Q, Pan J, Lei B, Huang A, Qi S: MIR517C inhibits autophagy and the epithelial-to-mesenchymal (-like) transition phenotype in human glioblastoma through KPNA2-dependent disruption of TP53 nuclear translocation. Autophagy 2015, 11(12):2213-2232.

15. Drucker E, Holzer K, Pusch S, Winkler J, Calvisi DF, Eiteneuer E, Herpel E, Goeppert B, Roessler S, Ori A et al: Karyopherin alpha2-dependent import of E2F1 and TFDP1 maintains protumorigenic stathmin expression in liver cancer. Cell Commun Signal 2019, 17(1):159.

16. Tang Z, Li C, Kang B, Gao G, Li C, Zhang Z: GEPIA: a web server for cancer and normal gene expression profiling and interactive analyses. Nucleic acids research 2017, 45(W1):W98-W102.

17. Rhodes DR, Yu J, Shanker K, Deshpande N, Varambally R, Ghosh D, Barrette T, Pandey A, Chinnaiyan AM: ONCOMINE: a cancer microarray database and integrated data-mining platform. Neoplasia 2004, 6(1):1-6.

18. Cerami E, Gao J, Dogrusoz U, Gross BE, Sumer SO, Aksoy BA, Jacobsen A, Byrne CJ, Heuer ML, Larsson $\mathrm{E}$ et al: The $\mathrm{cBio}$ cancer genomics portal: an open platform for exploring multidimensional cancer genomics data. Cancer discovery 2012, 2(5):401-404.

19. Gao J, Aksoy BA, Dogrusoz U, Dresdner G, Gross B, Sumer SO, Sun Y, Jacobsen A, Sinha R, Larsson E et al: Integrative analysis of complex cancer genomics and clinical profiles using the cBioPortal. Science signaling 2013, 6(269):pl1.

20. Tate JG, Bamford S, Jubb HC, Sondka Z, Beare DM, Bindal N, Boutselakis H, Cole CG, Creatore C, Dawson E et al: COSMIC: the Catalogue Of Somatic Mutations In Cancer. Nucleic acids research 2019, 47(D1):D941-D947.

21. Jezequel P, Frenel JS, Campion L, Guerin-Charbonnel C, Gouraud W, Ricolleau G, Campone M: bcGenExMiner 3.0: new mining module computes breast cancer gene expression correlation analyses. Database : the journal of biological databases and curation 2013, 2013:bas060.

22. Men C, Chai H, Song X, Li Y, Du H, Ren Q: Identification of DNA methylation associated gene signatures in endometrial cancer via integrated analysis of DNA methylation and gene expression systematically. J Gynecol Oncol 2017, 28(6):e83.

23. Shinawi T, Hill VK, Krex D, Schackert G, Gentle D, Morris MR, Wei W, Cruickshank G, Maher ER, Latif F: DNA methylation profiles of long- and short-term glioblastoma survivors. Epigenetics 2013, 8(2):149156.

24. Chandrashekar DS, Bashel B, Balasubramanya SAH, Creighton CJ, Ponce-Rodriguez I, Chakravarthi B, Varambally S: UALCAN: A Portal for Facilitating Tumor Subgroup Gene Expression and Survival Analyses. Neoplasia 2017, 19(8):649-658.

25. Ye J, Zhong L, Xiong L, Li J, Yu L, Dan W, Yuan Z, Yao J, Zhong P, Liu J et al: Nuclear import of NLSRARalpha is mediated by importin alpha/beta. Cellular signalling 2020:109567. 
26. Fan X, Li Z, Wang X, Wang J, Hao Z: Silencing of KPNA2 inhibits high glucose-induced podocyte injury via inactivation of mTORC1/p70S6K signaling pathway. Biochemical and biophysical research communications 2020, 521(4):1017-1023.

27. Song H, Song J, Lu L, Li S: SNHG8 is upregulated in esophageal squamous cell carcinoma and directly sponges microRNA-411 to increase oncogenicity by upregulating KPNA2. OncoTargets and therapy 2019, 12:6991-7004.

28. Klein A, Wessel R, Graessmann M, Jurgens M, Petersen I, Schmutzler R, Niederacher D, Arnold N, Meindl A, Scherneck S et al: Comparison of gene expression data from human and mouse breast cancers: identification of a conserved breast tumor gene set. International journal of cancer 2007, 121(3):683-688.

29. Wang JJ, Huang YQ, Song W, Li YF, Wang H, Wang WJ, Huang M: Comprehensive analysis of the IncRNAassociated competing endogenous RNA network in breast cancer. Oncology reports 2019, 42(6):2572-2582.

30. Winnepenninckx V, Lazar V, Michiels S, Dessen P, Stas M, Alonso SR, Avril MF, Ortiz Romero PL, Robert T, Balacescu $O$ et al: Gene expression profiling of primary cutaneous melanoma and clinical outcome. Journal of the National Cancer Institute 2006, 98(7):472-482.

31. Wang Cl, Wang CL, Wang CW, Chen CD, Wu CC, Liang Y, Tsai YH, Chang YS, Yu JS, Yu CJ: Importin subunit alpha-2 is identified as a potential biomarker for non-small cell lung cancer by integration of the cancer cell secretome and tissue transcriptome. International journal of cancer 2011, 128(10):2364-2372.

32. Krawczyk M, Liebe R, Lammert F: Towards genetic prediction of non-alcoholic fatty liver disease trajectories: PNPLA3 and beyond. Gastroenterology 2020.

33. Della Corte CM, Sen T, Gay CM, Ramkumar K, Diao L, Cardnell RJ, Rodriguez BL, Stewart CA, Papadimitrakopoulou VA, Gibson $L$ et al: STING pathway expression identifies non-small cell lung cancers with an immune-responsive phenotype. J Thorac Oncol 2020.

34. Rehman SU, Rizwan M, Khan S, Mehmood A, Munir A: Proteomic Analysis of Medicinal Plant Calotropis Gigantea by insilico Peptide Mass Fingerprinting. Curr Comput Aided Drug Des 2020.

35. Povedano E, Ruiz-Valdepenas Montiel V, Gamella M, Pedrero M, Barderas R, Pelaez-Garcia A, Mendiola M, Hardisson D, Feliu J, Yanez-Sedeno P et al: Amperometric bioplatforms to detect regional DNA methylation with single-base sensitivity. Anal Chem 2020.

36. Richardson AL, Wang ZC, De Nicolo A, Lu X, Brown M, Miron A, Liao X, Iglehart JD, Livingston DM, Ganesan S: X chromosomal abnormalities in basal-like human breast cancer. Cancer cell 2006, 9(2):121-132.

37. Ma XJ, Dahiya S, Richardson E, Erlander M, Sgroi DC: Gene expression profiling of the tumor microenvironment during breast cancer progression. Breast cancer research : BCR 2009, 11(1):R7.

38. Curtis C, Shah SP, Chin SF, Turashvili G, Rueda OM, Dunning MJ, Speed D, Lynch AG, Samarajiwa S, Yuan $Y$ et al: The genomic and transcriptomic architecture of 2,000 breast tumours reveals novel subgroups. Nature 2012, 486(7403):346-352. 


\section{Figures}

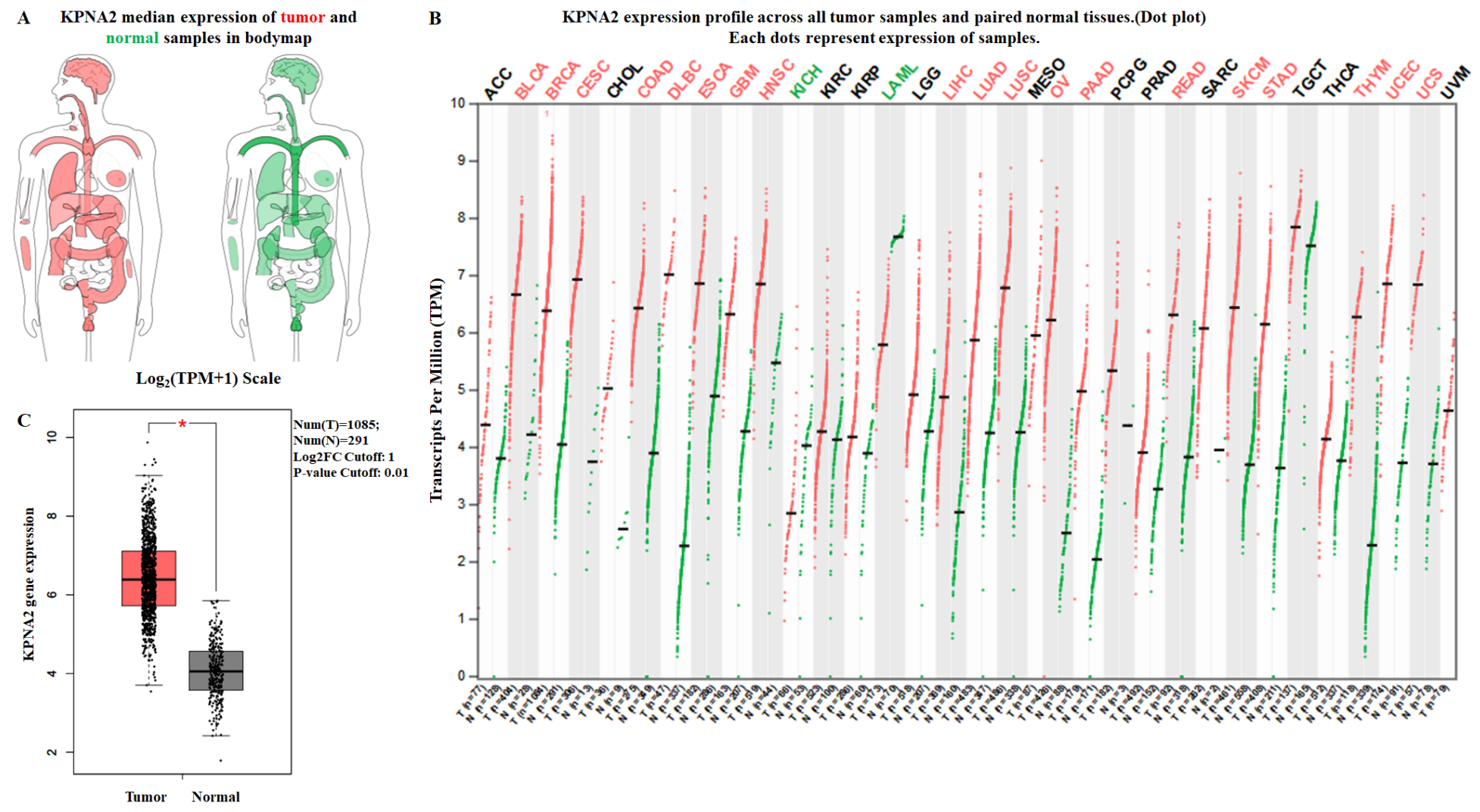

\section{Figure 1}

Expression of KPNA2 in breast cancer and normal tissues from GEPIA. (A) KPNA2 median expression of tumor (red) and normal (green) samples in bodymap; (B) KPNA2 expression profile across all tumor (red) and paired normal (green) tissues. Each dot represents the expression of sample. (C) The expression of KPNA2 mRNA in breast cancer tissues (red box) and paired normal tissues (black box) from GEPIA. 
A

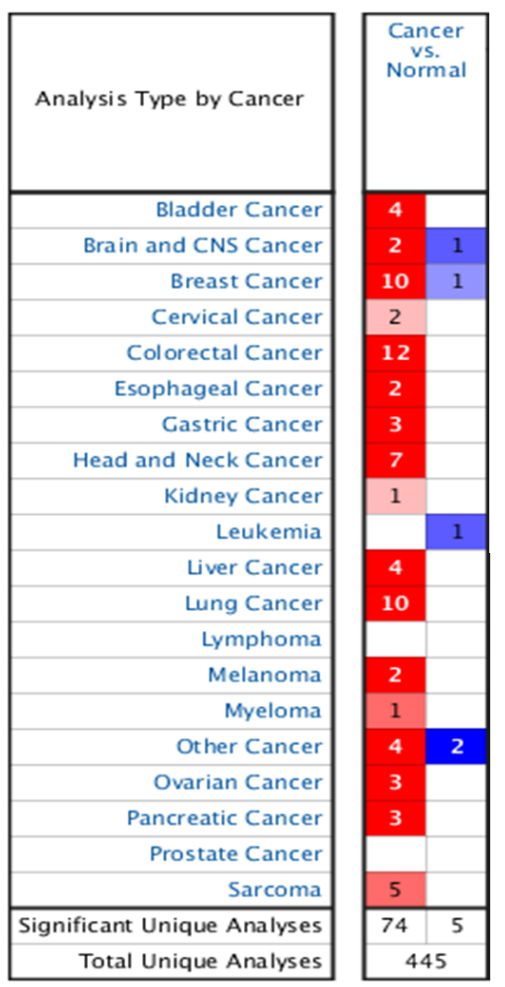

B
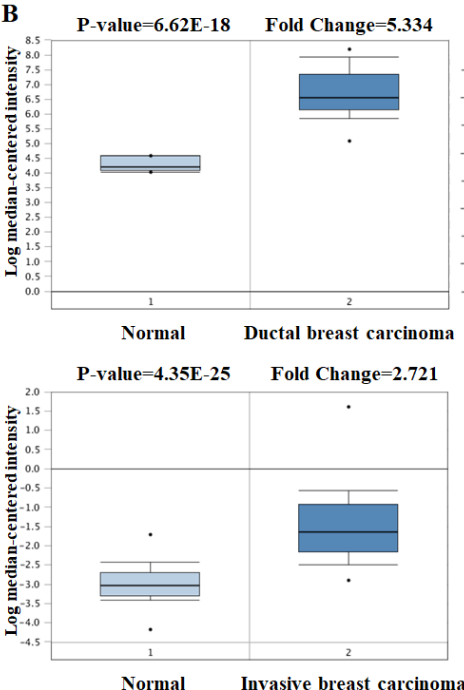

Normal Invasive breast carcino

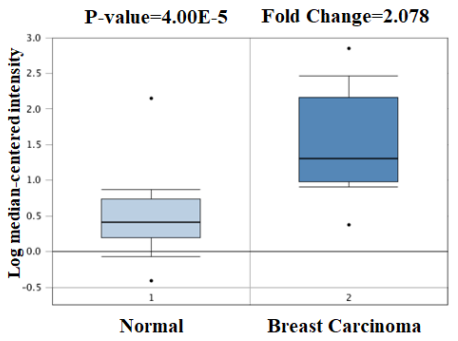

P-value $=1.84 \mathrm{E}-31 \quad$ Fold Change $=4.718$

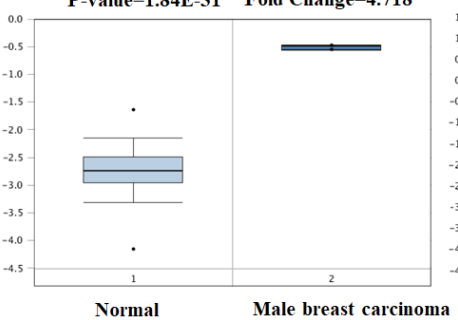

Male breast carcinomal

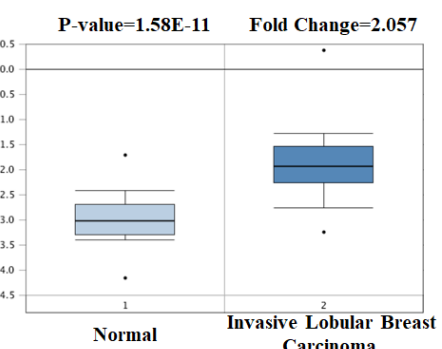

Carcinoma

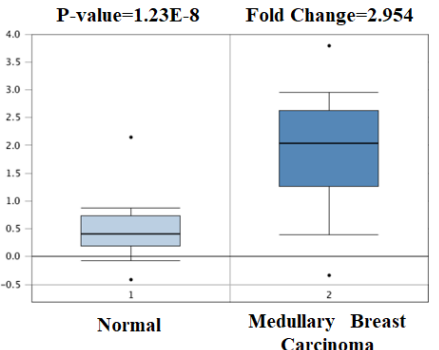
Carcinoma
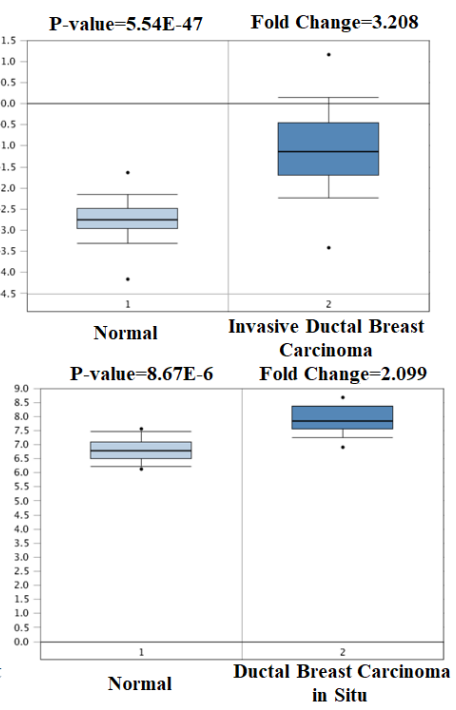

\section{Figure 2}

KPNA2 analysis in breast cancer (Oncomine database). (A) The online Oncomine analysis tool (red: overexpression, blue: down expression) was performed to compare KPNA2 expression levels in breast cancer specimens with matched normal specimens. The thresholds for significant probes for each microarray dataset included a two-fold difference in expression between cancer and normal samples and $\mathrm{P}<0.0001$. (B) The box plot compares KPNA2 expression in cancer samples (right) and matches normal (left) samples generated from the Oncomine database. 


\begin{tabular}{ll}
\hline Nonsense substitution & $0(0.00 \%)$ \\
\hline Missense substitution & $6(30.00 \%)$ \\
\hline Synonymous substitution $0(0.00 \%)$ \\
Inframe insertion & $0(0.00 \%)$ \\
Frameshift insertion & $0(0.00 \%)$ \\
Inframe deletion & $0(0.00 \%)$ \\
Frameshift deletion & $0(0.00 \%)$ \\
Complex mutation & $0(0.00 \%)$ \\
Other & $0(0.00 \%)$ \\
Total unique samples & 20
\end{tabular}

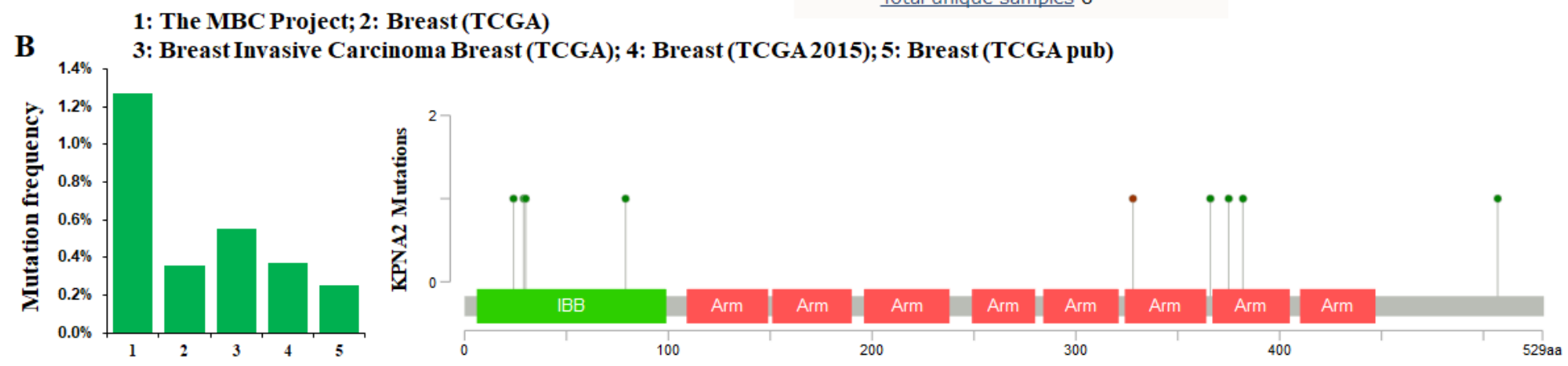

1: The MBC Project; 2: Breast (TCGA)

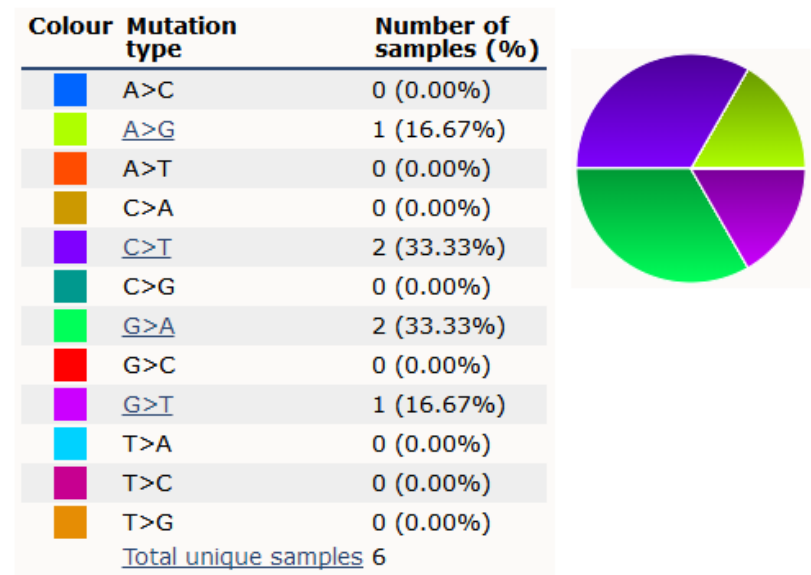

Figure 3

KPNA2 mutations in human breast cancer. (A) The pie chart generated by COSMIC summarizes the observed mutation types, including nonsense substitutions, missense substitutions, synonymous substitutions, inframe insertions, frameshift insertions, inframe deletions, frameshift deletions, and complex mutations. (B) As determined by cBioPortal, the KPNA2 mutation frequency was less than $2 \%$ in patients with breast cancer. 
Box and whisker plot of KPNA2 expression according to

(all DNA microarry data)

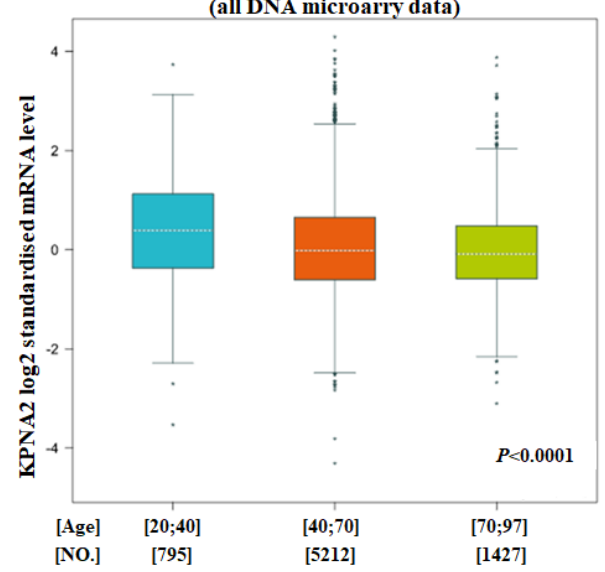

Box and whisker plot of KPNA2 expression according to

(all DNA microarry data)

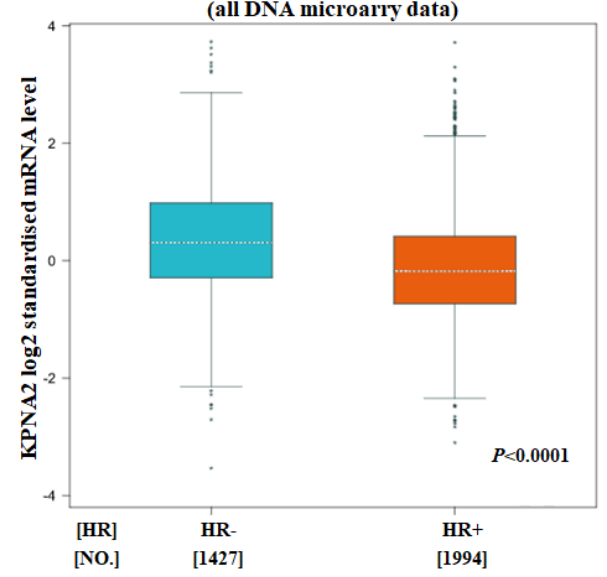

Box and whisker plot of KPNA2 expression according to (all DNA microarry data)

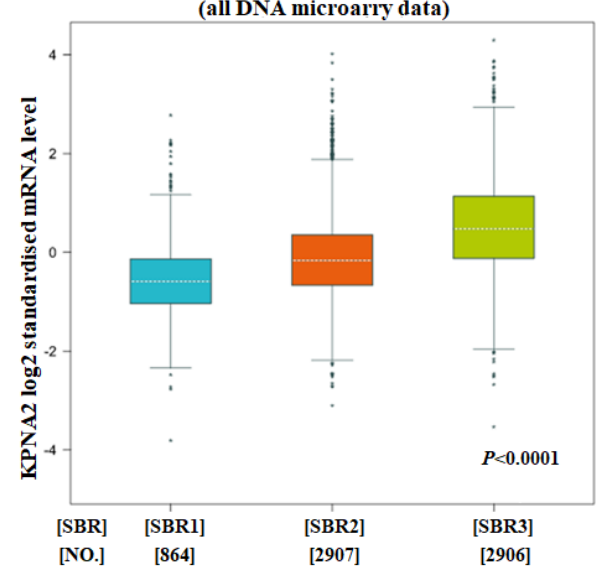

Box and whisker plot of KPNA2 expression according to

(all DNA microarry data)

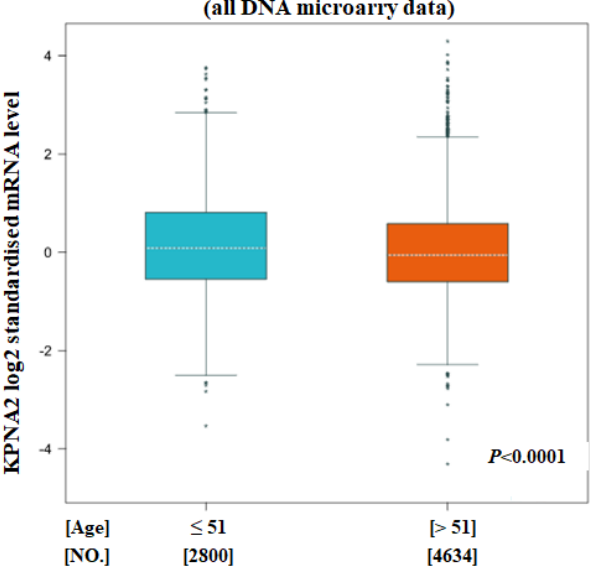

Box and whisker plot of KPNA2 expression according to HER2 (IHC)

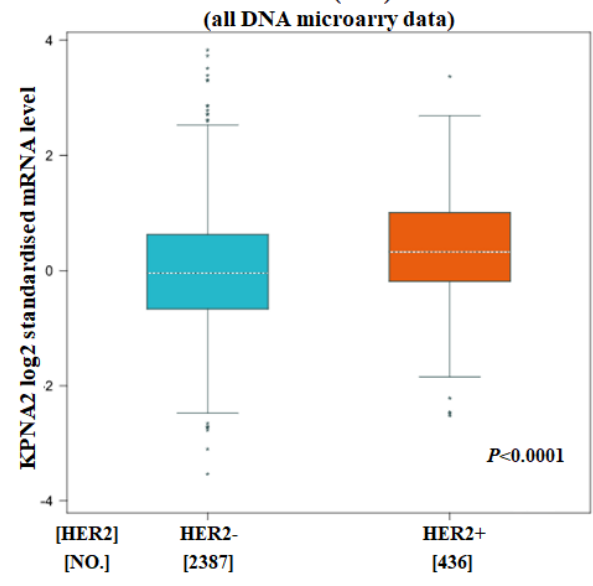

Box and whisker plot of KPNA2 expression according to triple-negative status (all DNA microarry data)

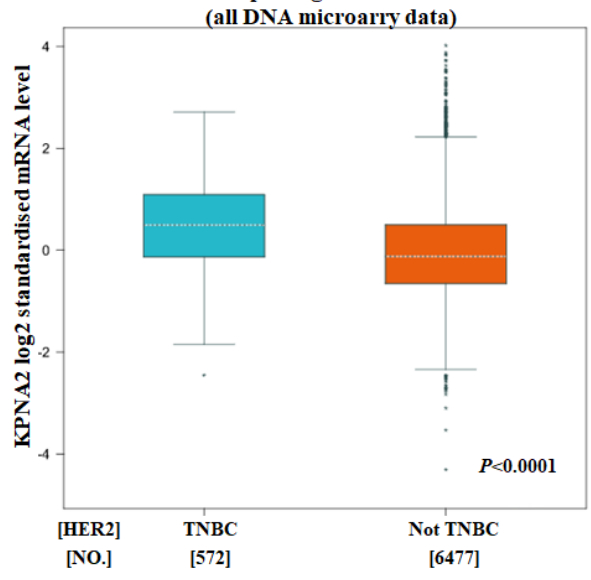

Box and whisker plot of KPNA2 expression according to ER (IHC)

(all DNA microarry data)

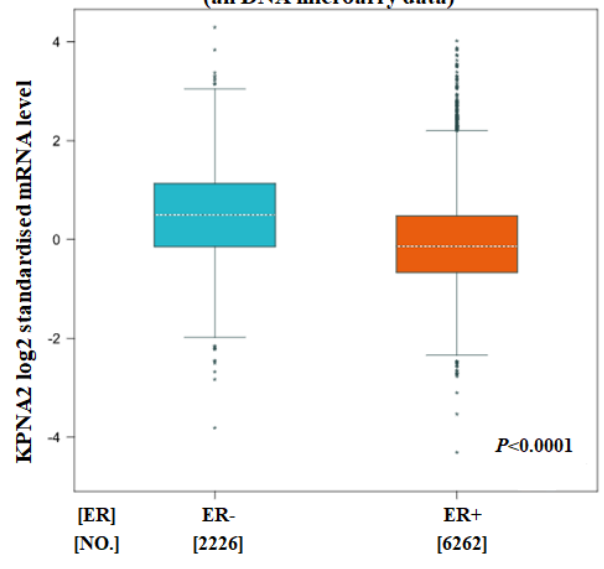

Box and whisker plot of KPNA2 expression according to nodal status (all DNA microarry data)

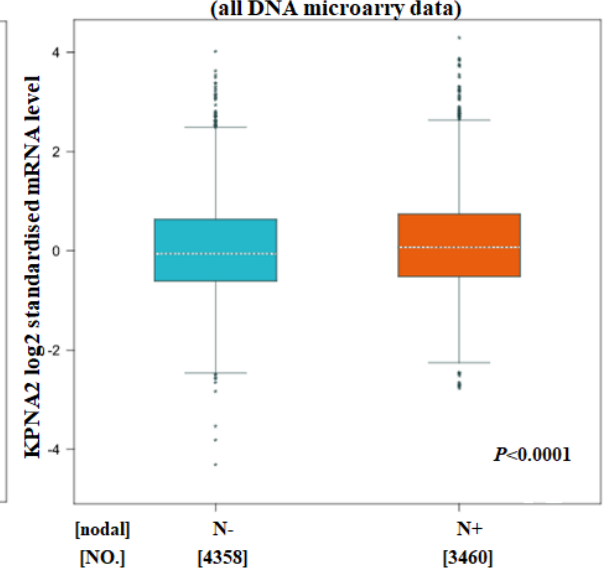

Box and whisker plot of KPNA2 expression according to Basal-like status (all DNA microarry data)

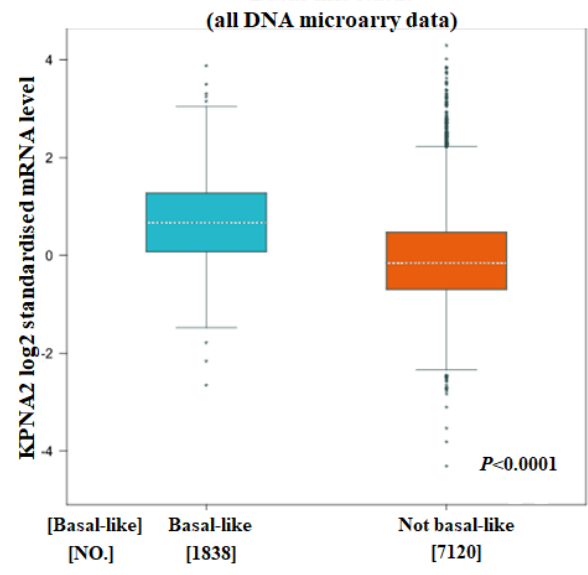

\section{Figure 4}

Genetic alterations in KPNA2 and clinicopathological parameters. Based on clinical pathology parameters, the expression profile of KPNA2 was expressed in the PAM50 breast cancer subtype using 5861 patients in bc-GenExMiner 4.4. A globally significant difference between the groups was assessed by Welch's t-test to generate $\mathrm{p}$-values, as well as the Dunnett-Tukey-Kramer test. 
A Top15 genes positively correlated to KPNA2 expression

\begin{tabular}{llrrr|}
$\begin{array}{l}\text { Correlated } \\
\text { Gene }\end{array}$ & Cytoband & $\begin{array}{c}\text { Spearman's } \\
\text { Correlation }\end{array}$ & p-Value & q-Value A \\
\hline TPX2 & $20 q 11.21$ & 0.832 & $3.87 e-256$ & $\mathbf{7 . 8 0 e - 2 5 2}$ \\
\hline CCNB2 & $15 q 22.2$ & 0.829 & $5.53 e-253$ & $\mathbf{5 . 5 8 e - 2 4 9}$ \\
CCNA2 & $4 q 27$ & 0.825 & $2.98 \mathrm{e}-248$ & $\mathbf{2 . 0 0 e - 2 4 4}$ \\
BIRC5 & $17 q 25.3$ & 0.823 & $8.38 \mathrm{e}-246$ & $\mathbf{4 . 2 3 e - 2 4 2}$ \\
CENPA & $2 \mathrm{p} 23.3$ & 0.819 & $4.83 e-241$ & $\mathbf{1 . 9 5 e - 2 3 7}$ \\
NCAPH & $2 \mathrm{q} 11.2$ & 0.814 & $4.48 \mathrm{e}-236$ & $\mathbf{1 . 4 4 e - 2 3 2}$ \\
CEP55 & $10 \mathrm{q} 23.33$ & 0.814 & $4.98 \mathrm{e}-236$ & $\mathbf{1 . 4 4 e - 2 3 2}$ \\
BUB1 & $2 \mathrm{q} 13$ & 0.813 & $2.42 \mathrm{e}-235$ & $\mathbf{6 . 1 1 e - 2 3 2}$ \\
AURKA & $20 \mathrm{q} 13.2$ & 0.811 & $2.94 \mathrm{e}-233$ & $\mathbf{6 . 6 0 e - 2 3 0}$ \\
KIF4A & Xq13.1 & 0.809 & $4.08 \mathrm{e}-231$ & $\mathbf{8 . 2 3 e - 2 2 8}$ \\
MCM10 & $10 \mathrm{p} 13$ & 0.805 & $1.43 \mathrm{e}-227$ & $\mathbf{2 . 6 3 e - 2 2 4}$ \\
KIF2C & $1 \mathrm{p} 34.1$ & 0.803 & $4.75 \mathrm{e}-225$ & $\mathbf{7 . 9 9 e - 2 2 2}$ \\
CDCA8 & $1 \mathrm{p} 34.3$ & 0.800 & $1.15 \mathrm{e}-222$ & $\mathbf{1 . 7 8 e - 2 1 9}$ \\
NCAPG & $4 \mathrm{p} 15.31$ & 0.799 & $5.62 \mathrm{e}-221$ & $\mathbf{8 . 1 0 e - 2 1 8}$ \\
PLK1 & $16 \mathrm{p} 12.2$ & 0.797 & $5.47 \mathrm{e}-219$ & $\mathbf{7 . 3 5 e - 2 1 6}$
\end{tabular}

B Correlation plot for all patients KPNA2 vs. TPX2

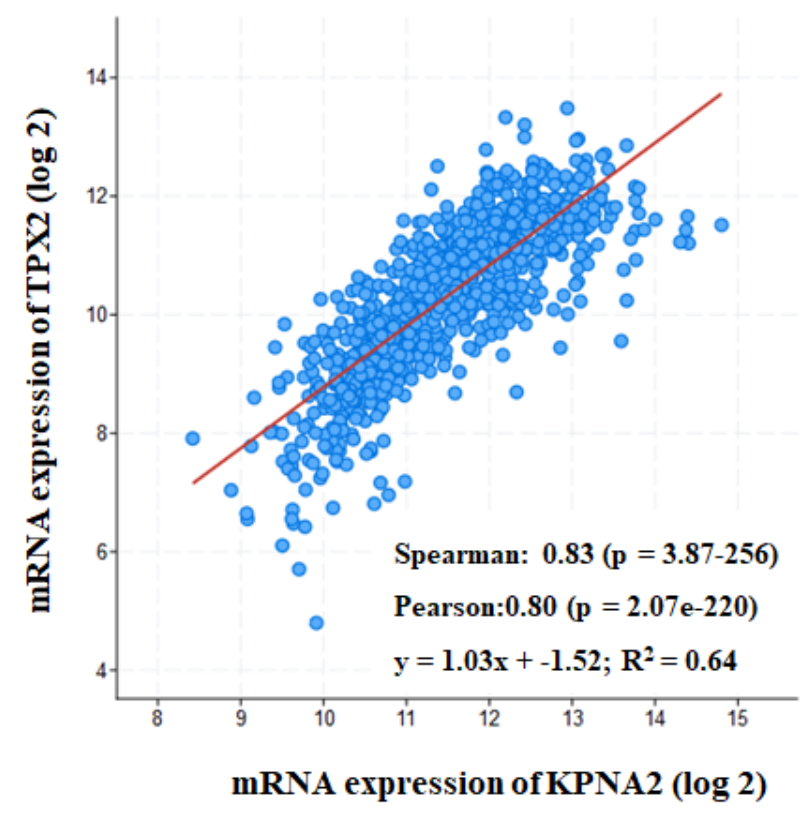

\section{Figure 5}

(A) Co-expression of the KPNA2 gene as determined by cBioPortal. (B) Regression analysis between KPNA2 and TPX2 in breast cancer performed by cBioPortal. 
KPNA2 (201088_at)

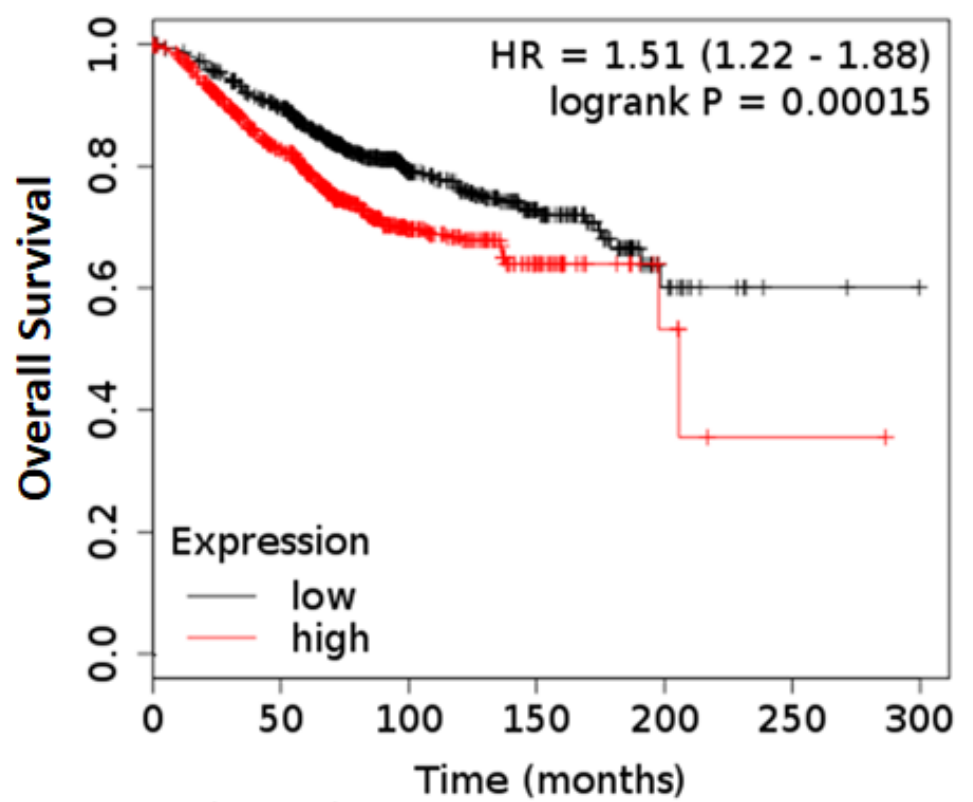

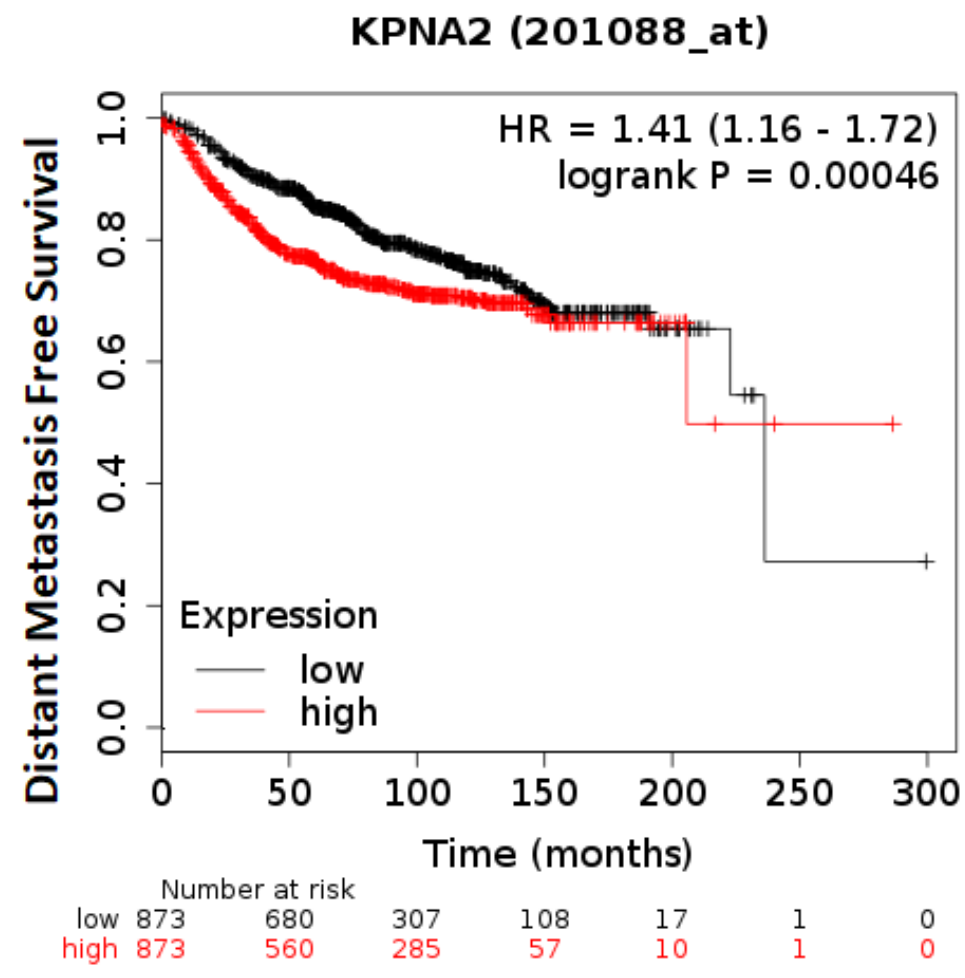
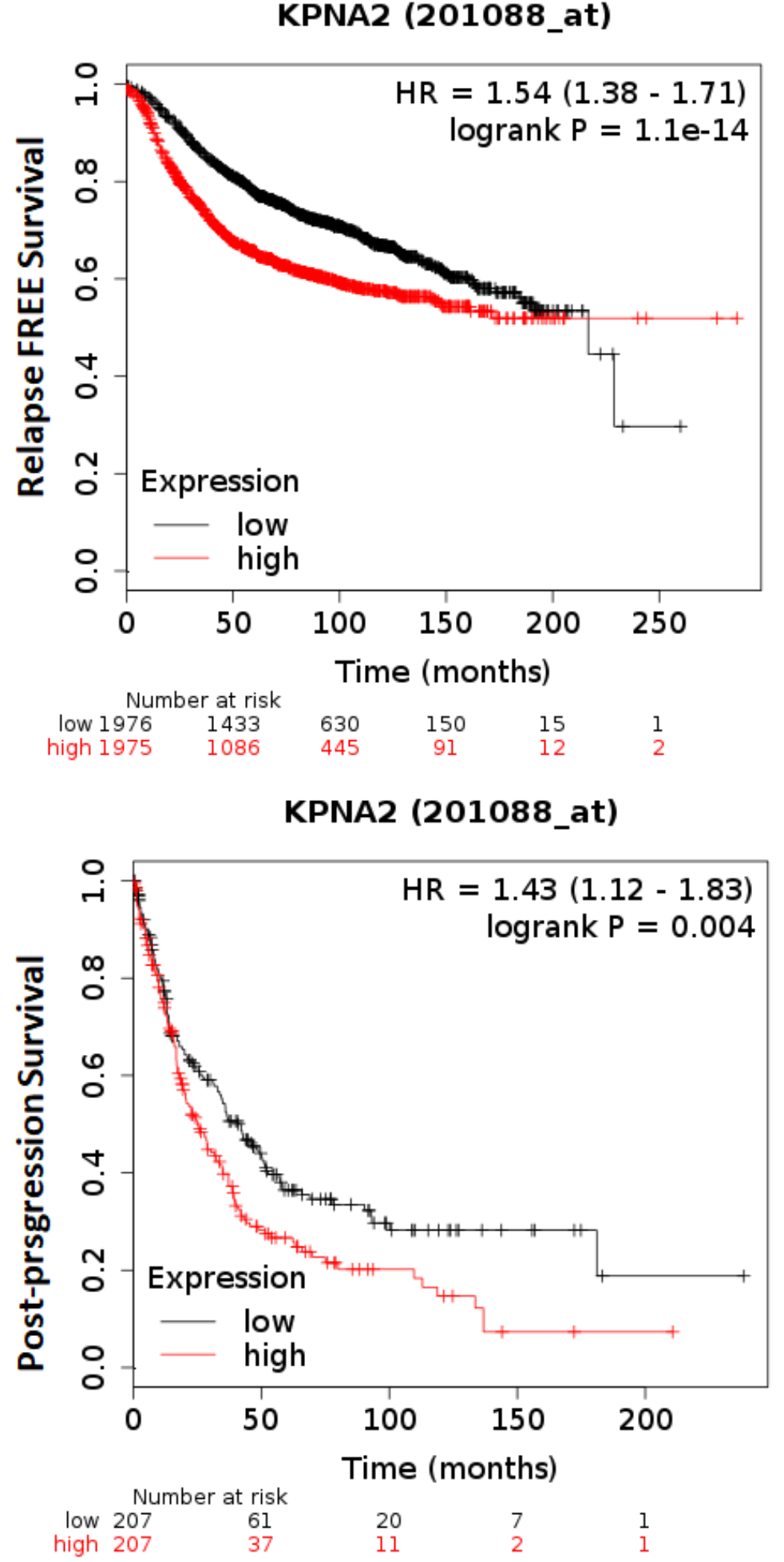

Figure 6

Relationship of KPNA2 expression and prognosis in breast cancers. OS (overall survival), RFS (relapse free survival), DMFS (distant metastasis free survival), and PPS (post-progression survival) curves calculated by Kaplan-Meier plotter for breast cancer patients, respectively. Survival probability is displayed on the $y$-axis, time (in months) on the x-axis. Black curves represent low KPNA2 expression, and red curves represent high KPNA2 expression. 

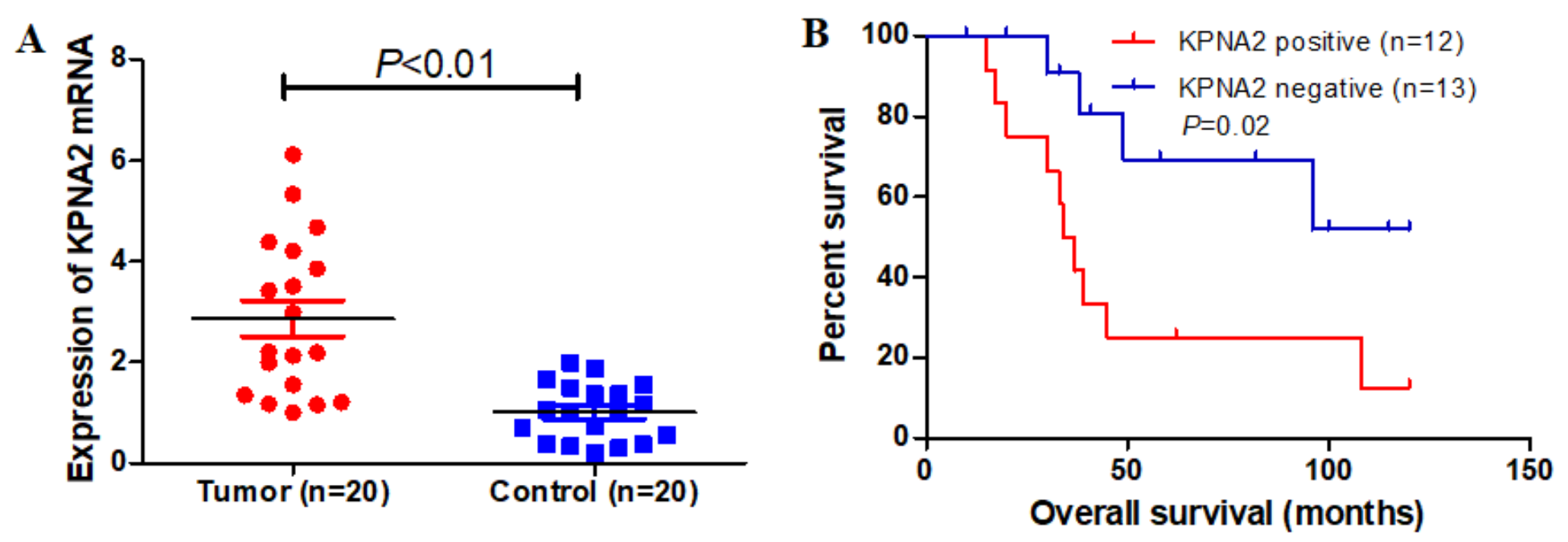

Figure 7

KPNA2 as a prognosis marker in breast cancer. (A) Expression of KPNA2 in tumor (20 cases) and adjacent normal mammary epithelium (20 cases). (B) Kaplan-Meier curves based on KPNA2 expression were drawn for overall survival in 25 patients.
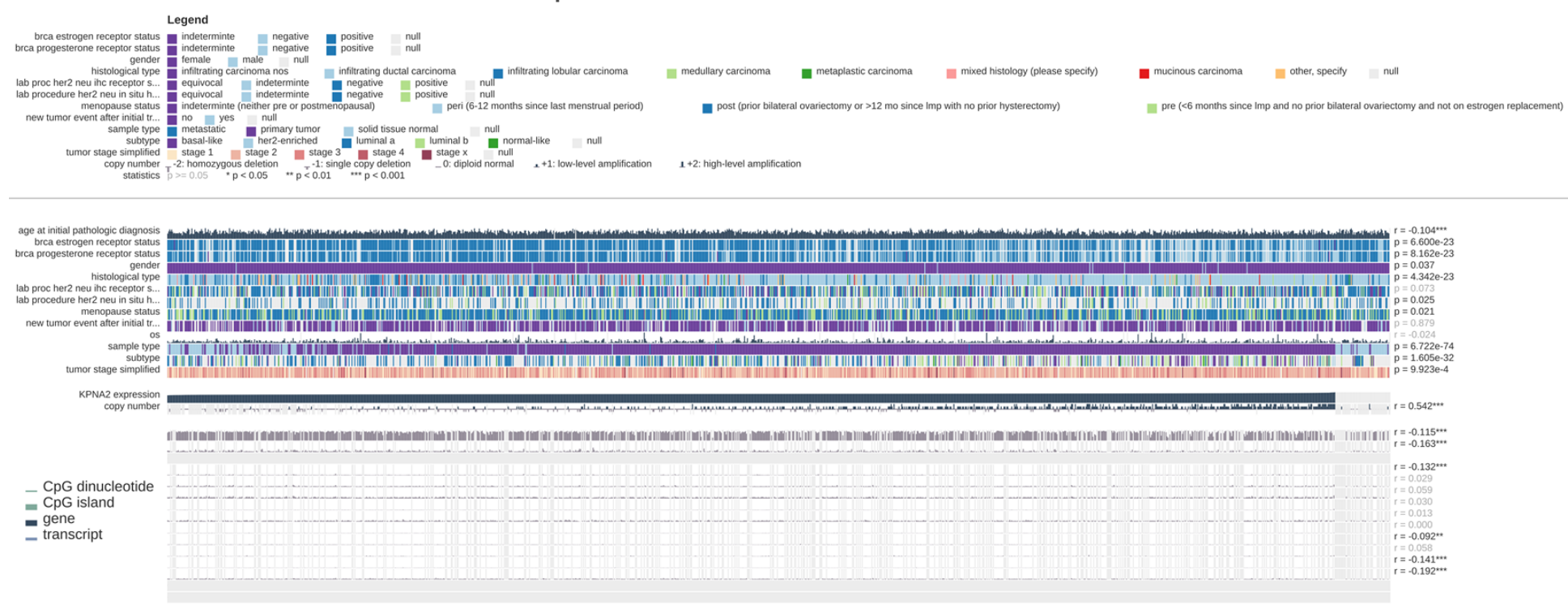

Figure 8

KPNA2 expression and methylation status in breast cancer using MEXPRESS tool. At the top of the figure, clinical TGCA data is displayed and classified according to KPNA2 expression. On the right side, the Pearson's correlation coefficient $r$ and $p$ values for Wilcoxon rank-sum test are displayed. The KPNA2 expression is represented by the orange line in the center of the graph. According to the expression of KPNA2, the highest expression was found on the left side and the lowest on the right side. The blue lines (lower right) represent the Infinium $450 \mathrm{k}$ probes linked to KPNA2. 


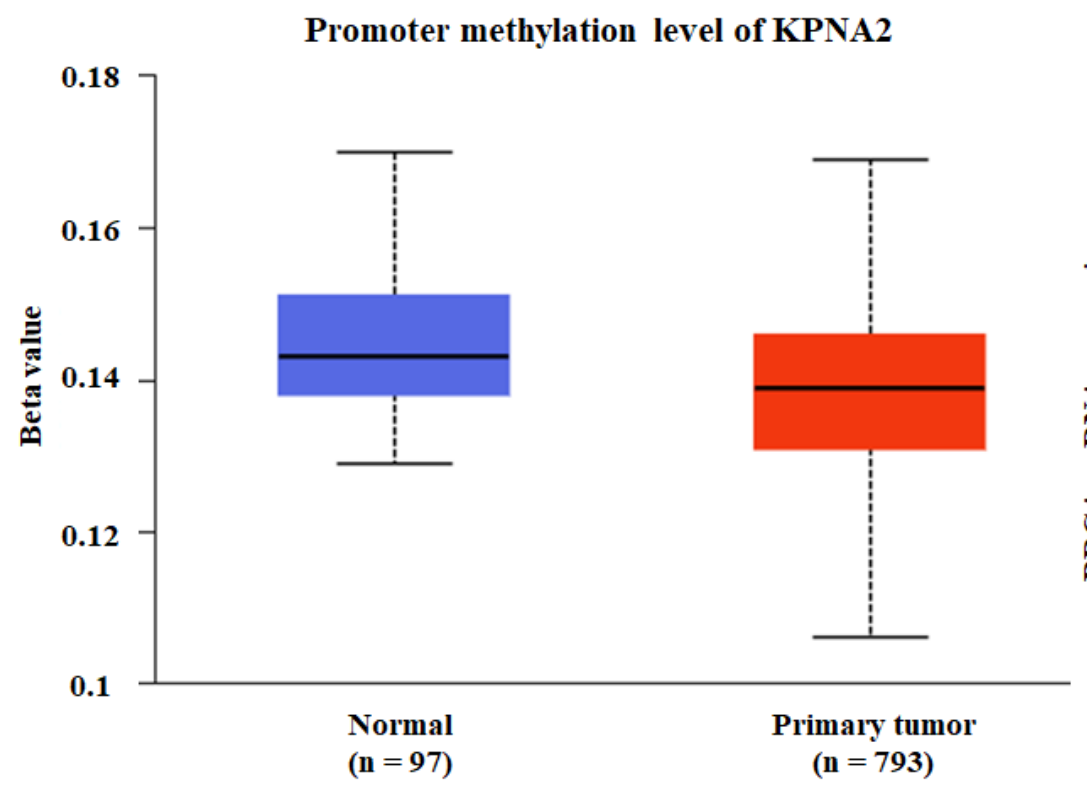

Correlation plot for all patients KPNA2 mRNA expression vs. Methylation level

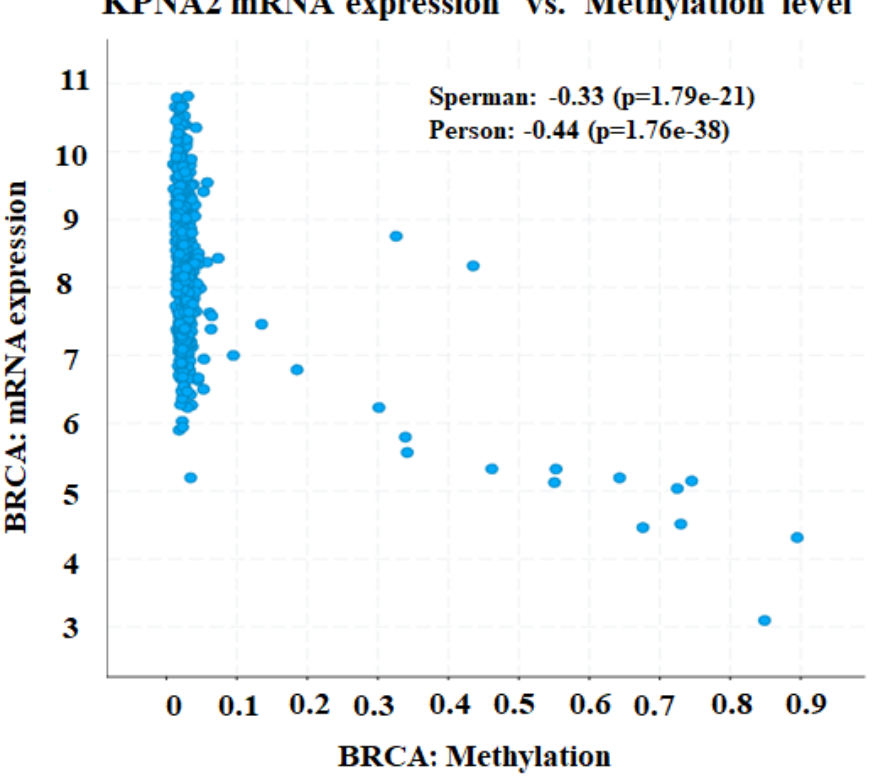

Figure 9

KPNA2 expression is upregulated by hypomethylation in breast tumors. (A) KPNA2 mRNA expression and promoter methylation in breast cancer. Box plot and $P$ value were produced using UALCAN. (B) Correlation of KPNA2 expression and promoter methylation was produced using cBioPortal. 

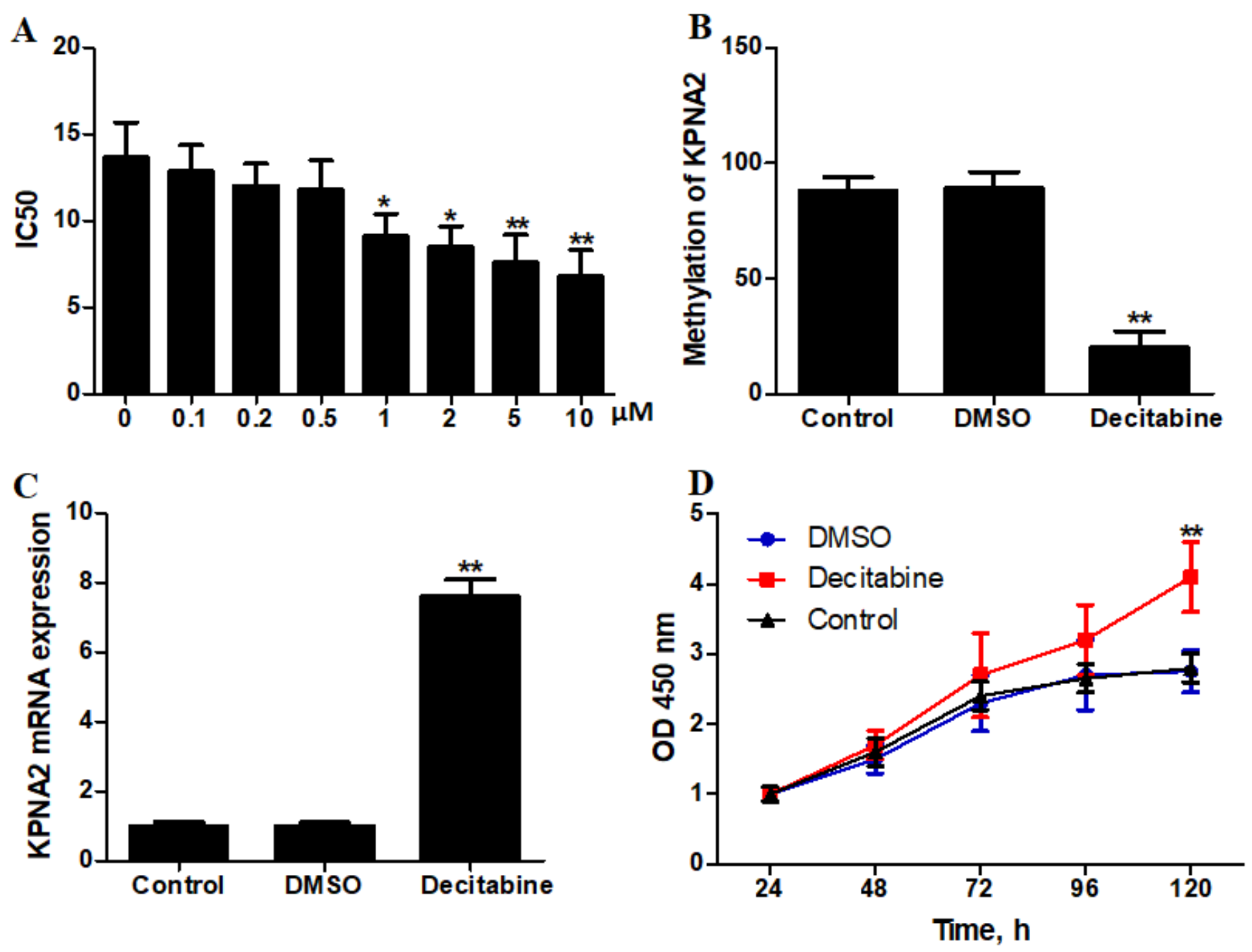

Figure 10

Decitabine treatment induced demethylation of KPNA2 in breast cancer cell line. (A) The minimum effective dose of decitabine was determined by CCK-8. (B) Decitabine treatment decreased KPNA2 methylation in the breast cancer cell line. (C) Restoration of KPNA2 mRNA by DNA methylation inhibitor Decitabine in breast cancer line. (D) Cellular proliferation was promoted by the upregulation of KPNA2 in the Decitabine group. 